\title{
Awake intubations are alive and well
}

\author{
Jonathan L. Benumof, MD
}

Received: 6 March 2015/ Accepted: 7 April 2015/Published online: 17 April 2015

(C) Canadian Anesthesiologists' Society 2015

To my mind, the early 1990 s marked the beginning of an era of consciousness in the worldwide anesthesia community to the practice of awake intubation to manage a recognized difficult airway (DA). At that time, the American Society of Anesthesiologists' (ASA) Task Force held meetings to create an ASA guideline on the management of the DA (ASA-DA-G). The impetus behind the creation of an ASA-DA-G was the finding that more than $85 \%$ of all respiratory-related closed malpractice claims involved a brain damaged or dead patient. ${ }^{1}$ In the very first meeting of the Task Force in 1990, the members realized that awake intubation for patients with recognized DAs would be a key part of the ASA-DA-G. They also understood that it would be prudent to publish a medical intelligence article that explained both the rationale for and the clinical practice of awake intubation, since the concept and practice of awake intubation might be unfamiliar to many practitioners. The subsequent medical intelligence article about the ASA-DA$\mathrm{G}$ was published in Anesthesiology in $1991,{ }^{2}$ and the ASADA-G itself was later published in 1993 . $^{3}$ Interestingly, a Canadian guideline on the management of the DA, which also recommends awake intubation for the patient with a known DA, was not published until $2013 .{ }^{4}$

The ASA-DA-G can be broken down into two fundamentally important concepts (i.e., pieces of advice) or limbs of an algorithm. First, if the practitioner recognizes that the airway will likely be difficult to manage, as revealed by the preoperative evaluation, then the airway should be secured by awake tracheal intubation.

J. L. Benumof, MD ( $₫)$

Department of Anesthesiology, UCSD Medical Center, 402

Dickinson Street - Mail Code 0801, San Diego, CA 92103, USA

e-mail: jbenumof@ucsd.edu
The logic of this limb of the ASA-DA-G is simple - the consequence of failing to recognize both the need for and the performance of an awake intubation in a patient with an anticipated DA is the development of a general anesthesia/paralysis-induced “can't intubate can't ventilate" situation. This often results in brain damage or outright death and contributes to the statistics cited above ${ }^{1}$ as well as many medicolegal lawsuits (personal observation). Conversely, it is not knowable whether a successfully performed awake intubation was actually necessary. In marginal, questionable, and otherwise unclear-as-to-what-to-do DA cases, awake intubation should be considered as the "better safe than sorry" therapeutic choice. The second important concept inherent in the ASA-DA-G is that the practitioner should have several effective "Plan Bs" immediately available in case general anesthesia is induced, with or without paralysis, and the airway is indeed difficult to manage.

This month's issue of the Journal features a study by Law et $a l .{ }^{5}$ which shows that there was no significant change in the use of awake tracheal intubation $(1.06 \%$ of 146,252 patients) at a large tertiary care centre in Canada over the studied years (2002-2013), even though there was a significant increase in the use of video laryngoscopy after the induction of general anesthesia over the same time period. The Law et al. study ${ }^{5}$ is a novel clear-cut scientific analysis of who is using which intubation technique for patients with a DA. The main result was not surprising. If the method of preoperative evaluation was relatively constant during the 2002-2013 period, which is a reasonable assumption, we would then expect the incidence of recognized DAs to be constant, which is what Law et al..$^{5}$ found. According to Law et al.,.$^{5}$ there was no intubation method (including video laryngoscopy) that was good enough (i.e., close to $100 \%$ effective) to replace 
and decrease the use of awake intubation for the patient with a recognized DA. In the Law et al. study, ${ }^{5}$ the increasing use of video laryngoscopy after the induction of general anesthesia must have been an elective "Plan A" for a few patients with a questionable DA or a "Plan B" for the unanticipated cannot intubate situation and not because it was a substitute for an awake intubation for the patient with a clearly recognized DA.

There are two main reasons why video laryngoscopy is not good enough to replace awake intubation for the patient with a recognized DA. First, there are many pathologic conditions that will predictably prevent success with video laryngoscopy and thereby contraindicate its use. Such conditions include a large cancerous mass, hematoma and abscess in the sight-line or application of the blade, major edema of the oropharynx and/or laryngopharynx, major distorting maxillary/mandibular fracture, the presence (or threat) of a large amount of blood that is difficult to clear, secretions, pus, edema fluid and gastric contents, a very compromised cervical spinal cord, and the predicted intolerance of apnea. Second, there are many anatomic configurations that will predictably prevent success with video laryngoscopy. Such configurations include a very large overbite of the maxillary teeth over the mandibular teeth, a large reduction in inter-incisor distance, a Mallampati class 4 oropharynx, a greatly decreased thyromental distance (as might be caused by severe micrognathia), significantly reduced mandibular space compliance (as might be caused by pre-existing mandibular space pathology), a very short thick neck, and the inability of the patient to assume a favourable position for tracheal intubation. In all of these pathological and anatomical conditions that result in a clearly recognized DA, awake intubation is indicated via either a flexible fibrescope (i.e., in the majority of conditions) or invasive/surgical methods in the neck (i.e., in the minority of conditions). These pathologic and anatomic considerations explain why the advent of video laryngoscopy cannot decrease the incidence of the use of awake intubation to manage the DA.

The $98.0 \%$ success rate of awake intubations achieved by the doctors at QEII Health Sciences Centre was outstanding. ${ }^{5}$ As someone who has performed many awake intubations over a long period of time for all sorts of reasons, I cannot stress enough that proper preparation of the patient is the key to success for an awake intubation. Furthermore, the key to success for proper preparation is psychological "buy in" by the patient needing the awake intubation. In addition, a dry airway is much easier to deal with than a wet airway; accordingly, glycopyrrolate is strongly advocated. Adequate and complete topicalization with the local anesthetic is always possible, and I spend time ensuring that is achieved. I sometimes perform nerve blocks (i.e., cranial nerves IX and X) if the anatomy is favourable and if the patient's cooperation is required following awake intubation (e.g., awake neurological examination in the patient with a compromised cervical spinal cord). That being said, I do use some sedation to alleviate the anxiety a patient may have, yet I keep the patient rational, oriented, and meaningfully responsive to commands.

The $2.0 \%$ complication rate in the study by Law et al. was very low. Most of the few complications listed by the authors were either unavoidable in a very large series (i.e., "it happens to everyone") and/or trivial in clinical importance (see Table 4 in Law et al.). ${ }^{5}$ Only two complications involving very few patients (5 of 1,554 patients) were clinically significant (i.e., "patient excessively sedated" and "inadvertent immediate extubation" $)^{5}$ and probably avoidable with better technique. Although not mentioned per se in the Law et al. study, ${ }^{5}$ the medicolegal implications are noteworthy. Indeed, I have participated as an expert witness in two medicolegal cases wherein the stomach was ruptured by insufflating oxygen down the working channel of a fibreoptic bronchoscope (FOB) while passing the FOB through the esophagus.

In conclusion, Law et al..$^{5}$ showed that there was a constant rate of utilization of awake intubation during 2002-2013 at the QEII Health Sciences Centre. This result is expected in view of the certain irreducible incidence of very obvious DAs due to both pathologic and anatomic reasons. Furthermore, the increasing use of video laryngoscopy over the same years was most likely the primary "Plan B" for the unanticipated DA. As shown by the Law et al. ${ }^{5}$ study, awake intubation can be performed with a high degree of success and a very low complication rate in very large numbers of patients over a long period of time. That being said, despite the fact that some of the 1,554 awake intubations they documented undoubtedly saved brains and lives, I am equally certain that some of the procedures were unknowingly unnecessary. To the extent that awake intubations steadily saved these brains and lives, awake intubations are alive and well.

\section{Les intubations vigiles se portent bien}

Selon moi, le début des années 1990 a marqué le début d'une ère de prise de conscience, dans la communauté mondiale de l'anesthésie, du rôle de l'intubation vigile pour prendre en charge des voies aériennes reconnues comme étant difficiles. À l'époque, le groupe de travail de l'American Society of Anesthesiologists (ASA) tenait des 
réunions afin de créer une directive de l'ASA sur la prise en charge des voies aériennes difficiles (ASA-DA-G en anglais, pour ASA Difficult Airway Guideline). L'élan ayant motivé la création de cette directive est venu de l'observation que plus de $85 \%$ de toutes les réclamations réglées en faute professionnelle liées aux voies respiratoires impliquaient un patient ayant subi des lésions cérébrales ou un décès. ${ }^{1}$ Lors de la toute première réunion de ce Groupe de travail, en 1990, les membres ont réalisé que l'intubation vigile des patients présentant des voies aériennes difficiles reconnues jouerait un rôle clé dans leur directive. Ils ont également compris qu'il serait prudent de publier un article de référence médical qui expliquerait à la fois pourquoi et comment réaliser une intubation vigile dans la pratique clinique, étant donné que le concept et la pratique de ce type d'intubation étaient peut-être mal connus de plusieurs praticiens. L'article de référence médical subséquent à propos cette directive a été publié dans la revue Anesthesiology en $1991,{ }^{2}$ et la directive de l'ASA elle-même a été publiée en $1993 .^{3}$ Il est intéressant de noter qu'une directive canadienne sur la prise en charge des voies aériennes difficiles, qui recommande également l'intubation vigile chez les patients présentant de voies aériennes difficiles connues, n'a été publiée qu'en 2013 . $^{4}$

La directive de l'ASA peut être divisée en deux concepts (ou conseils) fondamentalement importants, ou bras d'un algorithme. Premièrement, si le praticien estime, après avoir réalisé son évaluation préopératoire, que les voies aériennes seront probablement difficiles à prendre en charge, alors il faut les sécuriser en pratiquant une intubation trachéale vigile. La logique derrière ce bras de la directive est simple: la conséquence, si l'on n'identifie pas le besoin d'une intubation vigile ou qu'on ne la réussit pas chez un patient présentant des voies aériennes anticipées comme difficiles, est la création d'une situation où il devient «impossible d'intuber, impossible de ventiler» ('cannot intubate, cannot ventilate') induite par l'anesthésie générale / la paralysie. Une telle situation entraîne souvent des lésions cérébrales, voire le décès, et contribue dès lors aux statistiques citées ci-dessus ${ }^{1}$ ainsi qu'à bon nombre de poursuites médicolégales (observation personnelle). À l'inverse, il est impossible de savoir si une intubation vigile réussie était en fait nécessaire. Dans les cas de voies aériennes difficiles marginales, questionnables ou dans les cas où l'incertitude règne, il convient alors de considérer l'intubation vigile comme un choix thérapeutique de «mieux vaut prévenir que guérir». Le deuxième concept important inhérent à la directive de l'ASA est que le praticien devrait avoir plusieurs «plans $B$ » efficaces à sa disposition immédiate au cas où l'anesthésie générale est induite, avec ou sans paralysie, et que les voies aériennes s'avèrent effectivement difficiles à prendre en charge.

Ce numéro du Journal présente une étude de Law et coll. $^{5}$ qui montre qu'il n'y a pas eu de changement significatif dans le recours à l'intubation trachéale vigile (1,06\% de 146252 patients) dans un important centre de soins tertiaires canadien au cours de la période à l'étude (2002-2013) et ce, malgré une augmentation significative dans l'utilisation de la vidéolaryngoscopie après l'induction de l'anesthésie générale au cours de la même période. L'étude de Law et coll. ${ }^{5}$ est une analyse scientifique innovante qui décrit sans équivoque qui utilise quelle technique d'intubation pour les patients présentant des voies aériennes difficiles. Le résultat principal n'est pas surprenant. Si la méthode d'évaluation préopératoire était relativement constante au cours de la période allant de 2002 à 2013, ce qui est une hypothèse raisonnable, il serait alors probable que l'incidence de voies aériennes difficiles reconnues soit constante - ce qu'ont observé Law et coll. ${ }^{5}$ Selon eux, aucune méthode d'intubation (y compris la vidéolaryngoscopie) n'était assez efficace (c.-à-d. une efficacité proche de $100 \%$ ) pour remplacer et réduire l'utilisation de l'intubation vigile chez les patients présentant des voies aériennes difficiles reconnues. Dans l'étude de Law et coll., ${ }^{5}$ l'utilisation croissante de la vidéolaryngoscopie après l'induction de l'anesthésie générale a dû constituer un «plan $\mathrm{A}$ » non urgent chez certains patients présentant peut-être des voies aériennes difficiles ou un «plan $\mathrm{B}$ » lors de situations « impossible d'intuber» non anticipées. Mais cette modalité n'a pas remplacé l'intubation vigile chez les patients présentant clairement des voies aériennes difficiles reconnues.

Deux raisons principales expliquent pourquoi la vidéolaryngoscopie ne suffit pas à remplacer l'intubation vigile chez le patient présentant des voies aériennes difficiles reconnues. En premier lieu, il existe plusieurs pathologies qui entravent de manière prévisible la réussite de la vidéolaryngoscopie et qui, par conséquent, rendent son utilisation contre-indiquée. Parmi ces maladies, citons une importante masse cancéreuse, un hématome ou un abcès dans la ligne de visibilité ou d'application de la lame, un œdème majeur de l'oropharynx et/ou du laryngopharynx, une fracture maxillaire ou mandibulaire déformante majeure, la présence (ou la menace) d'une importante quantité de sang difficile à déplacer, des sécrétions, du pus, du liquide provenant d'un œdème et le contenu gastrique, une moelle épinière cervicale très compromise, et l'intolérance anticipée de l'apnée. Deuxièmement, plusieurs configurations anatomiques gênent de manière prévisible la réussite de la vidéolaryngoscopie. Une surocclusion importante des dents maxillaires par dessus les dents mandibulaires, une réduction majeure de la distance entre les incisives, un oropharynx de classe 4 sur 
l'échelle de Mallampati, une distance thyromentale considérablement réduite (telle que celle causée par une micrognathie grave), une compliance très réduite de l'espace mandibulaire (qui pourrait avoir été causée par une pathologie préexistante de l'espace mandibulaire), un cou très court et épais, et l'incapacité du patient à prendre une position favorable à l'intubation trachéale - voilà quelques-unes des configurations anatomiques pouvant entraver une bonne vidéolaryngoscopie. Dans le cas de toutes ces conditions pathologiques et anatomiques qui entravent clairement un accès sécuritaire aux voies aériennes, l'intubation vigile est indiquée. Elle peut être réalisée avec un fibroscope flexible (dans la plupart des conditions) ou à l'aide de méthodes invasives / chirurgicales dans le cou (dans la minorité des conditions). Ces considérations pathologiques et anatomiques expliquent pourquoi l'avènement de la vidéolaryngoscopie n'a pu réduire l'incidence d'utilisation de l'intubation vigile pour prendre en charge des voies aériennes difficiles.

Le taux de réussite de 98,0\% des intubations vigiles atteint par les médecins du QEII Health Sciences Centre, en Nouvelle-Écosse, est remarquable. ${ }^{5}$ Ayant personnellement réalisé de nombreuses intubations vigiles pendant une période prolongée et pour toutes sortes de raisons, $\mathrm{j}$ 'insiste encore et toujours sur le fait qu'une bonne préparation du patient est la clé de la réussite d'une intubation vigile. Plus encore, la clé du succès d'une bonne préparation réside dans un patient convaincu de la nécessité et du bien fondé de l'intubation vigile. En outre, il est bien plus facile de prendre en charge des voies aériennes sèches que mouillées; dès lors, on recommande fortement l'utilisation de glycopyrrolate. Il est toujours possible de réaliser une topicalisation adaptée et complète avec l'anesthésique local, et je prends toujours le temps de m'assurer du succès de cette technique. Je réalise parfois des blocs nerveux (soit les nerfs crâniens IX et X) si l'anatomie s'y prête et si la coopération du patient est nécessaire après l'intubation vigile (par ex., en cas d'examen neurologique éveillé chez le patient dont la moelle épinière cervicale est endommagée). Ceci dit, j'utilise un sédatif pour soulager l'anxiété que le patient pourrait ressentir, mais je m'assure de garder le patient rationnel, orienté et réactif aux instructions.

Le taux de complications de 2,0 \% que rapportent Law et coll. est très bas. La majorité des rares complications énumérées par les auteurs étaient soit inévitables dans une série d'une telle envergure («ça arrive à tout le monde ») et/ou insignifiantes d'un point de vue clinique (voir tableau 4 de l'étude de Law et coll.). ${ }^{5}$ Seules deux complications touchant un nombre minime de patients (5 patients sur 1554) avaient une importance clinique (« sédation excessive du patient» et « extubation immédiate involontaire $»)^{5}$ et auraient probablement pu être évitées avec une meilleure technique. Bien que cela ne soit pas mentionné en toutes lettres dans l'étude de Law et coll., ${ }^{5}$ les implications médicolégales méritent d'être relevées. En fait, j'ai participé comme témoin expert à deux cas médicolégaux dans lesquels l'estomac avait été perforé en insufflant de l'oxygène dans le canal fonctionnant d'un bronchoscope à fibres optiques en passant le bronchoscope à travers l'œsophage.

En conclusion, Law et coll. ont montré que le taux d'utilisation de l'intubation vigile était constant au cours de la période allant de 2002 à 2013 dans leur institution, le QEII Health Sciences Centre. Ce résultat était prévisible en regard de l'incidence irréductible et certaine de voies aériennes difficiles très évidentes pour des raisons pathologiques et anatomiques. De plus, l'utilisation croissante de la vidéolaryngoscopie au cours de la même période a très probablement constitué le principal «plan $\mathrm{B} »$ lors de voies aériennes difficiles non anticipées. Comme le montre l'étude de Law et coll., l'intubation vigile peut être réalisée avec un important taux de réussite et un très faible taux de complications chez un nombre considérable de patients et sur une période prolongée. Ceci dit, malgré le fait que certaines des 1554 intubations vigiles documentées ont indubitablement sauvé des cerveaux et des vies, je suis tout aussi certain que quelques-unes des interventions étaient, à l'insu des praticiens, non nécessaires. Dans la mesure où les intubations vigiles continuent de sauver des cerveaux et des vies, les intubations vigiles se portent bien.

Conflicts of interest None declared.

Conflit d'intérêt Aucun.

\section{References}

1. Caplan RA, Posner KL, Ward RJ, Cheney FW. Adverse respiratory events in anesthesia: a closed claims analysis. Anesthesiology 1990; 72: 828-33.

2. Benumof $J L$. Management of the difficult airway. With special emphasis on awake tracheal intubation. Anesthesiology 1991; 75: 1087-110.

3. Anonymous. Practice guidelines for management of the difficult airway. A report of the American Society of Anesthesiologists Task Force on Management of the Difficult Airway. Anesthesiology 1993; 78: 597-602.

4. Law JA, Broemling N, Cooper RM, et al. The difficult airway with recommendations for management - part 2 - the Anticipated Difficult Airway. Can J Anesth 2013; 60: 1119-38.

5. Law JA, Morris IR, Brousseau PA, de la Ronde S, Milne AD. The incidence, success rate, and complications of awake tracheal intubation in 1,554 patients over 12 years: an historical cohort study. Can J Anesth 2015; 62: this issue; DOI: 10.1007/s12630015-0387-y. 